\title{
Estudo do impacto das deficiências de saneamento básico sobre a saúde pública no Brasil no período de 2001 a 2009
}

\author{
Study of the impact of deficiencies of sanitation on public health in Brazil \\ from 2001 to 2009
Júlio César Teixeira', Guilherme Soares de Oliveira², Amanda de Mello Viali², Samuel Soares Muniz²

\begin{abstract}
RESUMO
O artigo teve como objetivo desenvolver um estudo do impacto sobre a saúde pública das deficiências do saneamento básico no Brasil no período de 2001 a 2009. Os óbitos resultantes de doenças relacionadas ao saneamento básico inadequado corresponderam, em média, a 13.449 mortes por ano, ou seja, cerca de 1,31\% do total. A média anual de casos de notificação compulsória devido a doenças relacionadas ao saneamento básico inadequado foi de 466.351 casos, com uma despesa de $\mathrm{R} \$$ 30.428.324,92 em consultas médicas nesse período. Foi identificada também uma média anual de 758.750 internações hospitalares devido a deficiências do saneamento básico, com uma despesa total de R 2.111.567.634,61 no período. A despesa total com consultas médicas e internações hospitalares devido a doenças associadas ao saneamento básico consumiu 2,84\% dos gastos do Sistema Único de Saúde nesse intervalo de tempo.
\end{abstract}

Palavras-chave: saneamento; saúde pública; óbitos; consultas; internações; indicadores.

\begin{abstract}
The objective of this article was to develop a study of the impact on public health of the sanitation deficiencies in Brazil from 2001 to 2009. Deaths due to diseases related to inadequate sanitation accounted, on average, 13,449 deaths per year, or about $1.31 \%$ of total deaths. The annual average of compulsory notification of cases due to diseases related to inadequate sanitation conditions was 466,351 cases, with an expense of $\mathrm{R} \$ 30,428,324.92$ with medical appointments in this period. It was identified an average of 758,750 hospitalizations due to lack of sanitation, with a total expenditure of $\mathrm{R} \$ 2,111,567,634.61$ in the period. The total expenditure on medical appointments and hospitalizations due to diseases associated with sanitation consumed $2.84 \%$ of the Unified Health System expenditures with these items in the period.
\end{abstract}

Keywords: sanitation; public health; deaths; medical appointments; hospitalizations; indicators..

\section{INTRODUÇÃO}

A infraestrutura sanitária deficiente desempenha uma interface com a situação de saúde e com as condições de vida das populações dos países em desenvolvimento, onde as doenças infecciosas continuam sendo uma importante causa de morbidade e mortalidade. A prevalência destas doenças constitui um forte indicativo de fragilidade dos sistemas públicos de saneamento (DANIEL et al., 2001).
No entanto, na mensuração das condições de vida e saúde persiste um desafio. A saúde deve ser pensada como uma resultante das relações entre as variáveis ambientais, sociais e econômicas que pressionam as condições de vida. Logo, em toda análise da situação da saúde, os indicadores básicos de desenvolvimento humano assumem uma importância fundamental, pois documentam as condições de vida da população e dimensionam o espaço social em que ocorrem as mudanças em seu estado (OPAS, 2007).

'Engenheiro Civil e de Segurança no Trabalho. Doutor em Saneamento, Meio Ambiente e Recursos Hídricos pela Universidade Federal de Minas Gerais (UFMG). Professor associado do Departamento de Engenharia Sanitária e Ambiental da Universidade Federal de Juiz de Fora (UFJF) - Juiz de Fora (MG), Brasil. Estudante de Engenharia Sanitária e Ambiental pela UFJF. Bolsista de Iniciação Científica - Juiz de Fora (MG), Brasil. Endereço para correspondência: Júlio César Teixeira - Departamento de Engenharia Sanitária e Ambiental da Faculdade de Engenharia da Universidade Federal de Juiz de Fora - Sala 4.103 - 4a Plataforma do Setor de Tecnologia - 36036-330 - Juiz de Fora (MG), Brasil - E-mail: juliotei@terra.com.br Recebido: 17/11/11 - Aceito: 25/10/13 - Reg. ABES: 133 
A população do Brasil, que chegou a 190,7 milhões de pessoas em 2010, cresce no menor ritmo já registrado ( $1,12 \%$ ao ano) e de maneira desigual pelo território, com as maiores taxas concentradas nas regiões Norte e Centro-Oeste. Ademais, a população brasileira cresceu 12,3\% desde 2000, quando havia 169,8 milhões de habitantes no País. Neste período, seguindo tendência das últimas décadas, a população rural perdeu 2 milhões de pessoas e reduziu sua participação para 15,6\% do total da população brasileira. Já a população urbana ganhou 23 milhões de habitantes e representa $84,4 \%$ do total dos brasileiros (IBGE, 2011).

Por outro lado, o processo de urbanização brasileiro se deu de forma muito desigual, isto é, com grandes diferenças entre classes sociais e entre regiões. Logo, os estados brasileiros são, em geral, muito desiguais em relação ao saneamento básico. Isso tem criado problemas dentro das cidades brasileiras, com informalidade na moradia e na infraestrutura de saneamento básico. A necessidade de racionar água existe em 25\% dos municípios da Região Norte e em 40\% dos municípios da Região Nordeste, ao passo que na Região Sudeste o problema é praticamente inexistente (IBGE, 2010).

No ano de 2007, foi promulgada a Lei Nacional de Saneamento Básico, $n^{\circ} 11.445$ (BRASIL, 2007), que estabelece que os serviços públicos de saneamento básico devem ser prestados com base em alguns princípios fundamentais, destacando-se a universalização do acesso aos serviços. A lei $\mathrm{n}^{\circ} 11.445$ aborda as especificidades de cada um dos serviços de saneamento, tal como definidos em lei: abastecimento de água; esgotamento sanitário; limpeza urbana e manejo de resíduos sólidos; drenagem e manejo das águas pluviais urbanas. Entretanto, o faz sob a perspectiva da integralidade aplicada ao saneamento básico e à integração desses serviços com outras políticas públicas que se relacionam mais diretamente com o seu campo de intervenção (BRASIL, 2009).

Em 2008, apesar de ser a sétima economia do mundo, o Brasil apresentava índices de cobertura de saneamento básico de países subdesenvolvidos. A Pesquisa Nacional de Saneamento Básico 2008 (IBGE, 2010) mostrou, por exemplo, que somente $46 \%$ dos domicílios no país estavam conectados a redes de esgotamento sanitário. Tais redes estavam presentes, principalmente, nos grandes e médios centros urbanos e nas sedes dos municípios com população acima de 50 mil habitantes. No entanto, as estatísticas oficiais consideram que se um município oferece algum serviço público de saneamento básico, isso já o inclui entre os municípios atendidos, independentemente da qualidade do serviço prestado e do percentual de cobertura populacional. Além disso, em 2008, aproximadamente $20 \%$ da população brasileira não dispunha de rede geral de abastecimento de água; 50\% dos municípios brasileiros depositavam o lixo a céu aberto, além de carências na área de drenagem urbana que geram inundações em cidades por todo o país.

Em 2009, o gasto público com saúde - União, estados e municípios - correspondeu a 3,86\% do Produto Interno Bruto do país, um valor aproximado de $\mathrm{R} \$ 125,1$ bilhões de reais. Inclusive, no Brasil, a parcela do orçamento federal destinada à saúde, excluídos gastos de estados e municípios, foi de 7,6\% do total de gastos da União (DATASUS, 2011), sendo inferior à média dos países africanos $(10,6 \%)$ e à média mundial (11,7\%) entre 2000 e 2009 (OMS, 2010).

Costa et al. (2010) afirmaram que o número médio de óbitos por doenças relacionadas a um saneamento inadequado no Brasil foi de 14.995 por ano no período 1996 a 1999, ou seja, 1,90\% do total de óbitos no País no período.

Para a Organização Mundial da Saúde (OMS, 2007), cerca de 233 mil pessoas morriam todo ano no Brasil por exposição a fatores de risco ambiental, como poluição do ar, água não tratada e falta de infraestrutura urbana, sendo que $19 \%$ de todas as mortes no país poderiam ser evitadas se fossem adotadas políticas públicas eficientes. A pesquisa levou em consideração as condições enfrentadas pelos brasileiros em seu dia a dia, afirmando que a poluição do ar causava óbito de 12,9 mil pessoas por ano e, ainda, com $22 \%$ das pessoas vivendo abaixo da linha da pobreza, a falta de água tratada e de esgotamento sanitário eram responsáveis pela morte de 15 mil brasileiros por ano.

Neste contexto, o objetivo do presente artigo é desenvolver um estudo sobre o impacto sobre a saúde pública das deficiências dos serviços de saneamento básico no país no período de 2001 a 2009.

\section{METODOLOGIA}

\section{Área geográfica e população de estudo}

A área geográfica de estudo foi todo o território brasileiro. A população estudada compreende a população brasileira que chegou a 190,7 milhões de pessoas em 2010, cuja taxa de crescimento anual atingiu 1,12\% ao ano na última década (IBGE, 2011).

\section{Universo de estudo}

O estudo epidemiológico das doenças relacionadas ao saneamento básico inadequado teve dois universos distintos. O primeiro, da mortalidade, dimensionado por meio de declarações de óbito, e o segundo, da morbidade, relacionada a casos de doenças de notificações compulsórias e de autorizações para internações hospitalares devido a doenças relacionadas ao saneamento básico inadequado.

\section{Base de dados}

A base de dados foi composta por elementos secundários provenientes do banco de dados Indicadores e Dados Básicos (BRASIL, 2011), disponível no sítio do Departamento de Informática do SUS (DATASUS, 2011), mantido pelo Ministério da Saúde e também por informações do Instituto Brasileiro de Geografia e Estatística - IBGE (2011), a saber:

- Censo Demográfico 2010;

- Sistema de Informações sobre Mortalidade (SIM);

- Sistema de Informação de Agravos de Notificação (SinanWeb); 
- $\quad$ Sistema de Informações Hospitalares (SIH);

- Sistema de Informações Ambulatoriais (SIA).

\section{Doenças estudadas}

Utilizou-se, para coleta dos dados da pesquisa, um grupo de doenças infecciosas e parasitárias, bem como um grupo de doenças infecciosas intestinais; ambos definidos pela Organização Mundial da Saúde (OMS) como capítulo e categoria, respectivamente, da Classificação Internacional de Doenças (OMS, 1997) (Quadro 1), segundo metodologia proposta por Costa et al. (2010).

\section{Gastos federais em saúde}

\section{devido à falta de saneamento}

Neste trabalho, os gastos federais em saúde devido a deficiências do saneamento básico foram apurados a partir de dois tipos de despesas:

1. gastos do Sistema Único de Saúde (SUS) com atendimento ambulatorial devido a doenças de notificação compulsória relacionadas ao saneamento básico inadequado;
2. gastos do SUS com morbidade hospitalar por doenças relacionadas ao saneamento básico inadequado.

\section{Análise dos dados}

A análise dos dados foi desenvolvida segundo um processo evolutivo, em etapas, de forma a permitir a determinação progressiva dos agravos relacionados ao saneamento básico inadequado. Tal processo envolveu, em sequência, as seguintes atividades:

- Criação de um diretório para cada sistema — SIM, SinanWeb, SIH e SIA - para onde foram transferidos, respectivamente, os arquivo de óbitos por residência, de doenças de notificação compulsória, de arquivos de autorizações de internações hospitalares e de informações ambulatoriais.

- Criação dos arquivos de definição contendo os critérios para tabulação e observando os grupos de doenças selecionados e os respectivos códigos da CID-10, constantes do Quadro 1.

- Tabulação dos dados via TabWin versão 2.0 e exportação para o Microsoft Office Excel 2007, para construção de tabelas.

Quadro 1 - Doenças relacionadas ao saneamento básico inadequado.

\begin{tabular}{|c|c|c|}
\hline Categoria & Doenças & CID-10 (1) \\
\hline \multirow{5}{*}{ 1. Doenças de transmissão feco-oral } & & $\mathrm{AOO}$ \\
\hline & Diarreias & $\mathrm{AO2-O4;}$ \\
\hline & & A06-A09 \\
\hline & Febres entéricas & $\mathrm{AO1}$ \\
\hline & Hepatite A & $\mathrm{B} 15$ \\
\hline \multirow{6}{*}{ 2. Doenças transmitidas por inseto vetor } & Dengue & A90-A91 \\
\hline & Febre amarela & A95 \\
\hline & Leishmanioses & B55 \\
\hline & Filariose linfática & B74 \\
\hline & Malária & B50-B54 \\
\hline & Doença de Chagas & B57 \\
\hline \multirow{2}{*}{ 3. Doenças transmitidas pelo contato com a Água } & Esquistossomose & B65 \\
\hline & Leptospirose & A27 \\
\hline \multirow{5}{*}{ 4. Doenças relacionadas com a higiene } & Doença dos olhos: & \\
\hline & Tracoma & A71 \\
\hline & Conjuntivites & $\mathrm{H} 1 \mathrm{O}$ \\
\hline & Doenças de pele: & \\
\hline & Micoses superficiais & B35-B36 \\
\hline \multirow{5}{*}{ 5. Geohelmintos e teníases } & & B68 \\
\hline & Helmintíases & B69 \\
\hline & & B71 \\
\hline & & B76-B83 \\
\hline & Teníases & B68-B69 \\
\hline
\end{tabular}

(1)CID-10: Classificação Internacional de Doenças. Revisão 1996 (OMS, 1997). 


\section{RESULTADOS E DISCUSSÃO}

\section{Mortalidade por doenças relacionadas ao saneamento básico inadequado}

Inicialmente, em relação ao estudo da mortalidade no Brasil existem, no dizer de Carvalho (1997), dois pontos importantes a serem considerados:

1. Há grande subnotificação de óbitos;

2. Há acentuado número de óbitos que tem como causa básica, registrada nos atestados de óbitos, sinais e sintomas mal definidos.

Estes dois problemas ainda são importantes nas regiões Norte e Nordeste do País, embora ao longo dos anos venham apresentando declínio (COSTA et al., 2010).

Os óbitos por doenças infecciosas e parasitárias (ODIP) representaram em média, no período de 2001 a 2009, cerca de 4,51\% do total de óbitos no país. Observa-se, ainda, que neste período, houve uma variação de 4,68 para 4,26\% no percentual de óbitos decorrentes dessas doenças em relação ao total de óbitos no Brasil (Tabela 1), confirmando uma tendência que vem sendo observada desde o século XX.

Observa-se, na Tabela 1, que os óbitos por causas definidas relacionadas ao saneamento básico inadequado (ORSBCD) corresponderam, em média, a cerca de 12.068 mortes por ano, entre 2001 e 2009.

Na Tabela 2, foi levantado, para cada ano do estudo, o número de óbitos por causas não definidas (OCND), ou seja, as mortes incluídas no Capítulo XVIII - "Sintomas, sinais e achados anormais de exames clínicos e de laboratório não classificados em outra parte" da Classificação Internacional de Doenças (OMS, 1997, p. 817).
A partir do número de OCND e do percentual de ORSBCD, estimou-se para cada ano o número de óbitos por causas não definidas que pode ser associado a deficiências do saneamento básico (ORSBND).

Observou-se que o número total de óbitos por doenças relacionadas ao saneamento básico inadequado (ORSB) variou de 14.460, em 2001, a 12.056 óbitos, em 2009, sendo que o valor médio encontrado foi de 13.449 mortes por ano ao longo dos anos 2000, ou seja, uma média de 1,31\% dos óbitos ocorridos no período de estudo. Observou-se ainda um número decrescente de mortes por doenças relacionadas ao saneamento básico inadequado no período de 2001 a 2009, com uma queda de $16,63 \%$.

Já na Tabela 3, é apresentada a distribuição da frequência de óbitos por causas definidas relacionadas ao ORSBCD no período de 2001 a 2009. Para facilitar a visualização, optou-se por apresentar os números referentes aos anos ímpares do período estudado.

Pôde-se verificar o aumento expressivo do número de mortes devido à dengue. No ano de 2001, foram registrados 42 óbitos, enquanto em 2009 , este número saltou para 375 , ou seja, durante este período, houve um aumento de $792 \%$ nos óbitos relacionados à doença.

As diarreias e a doença de Chagas representaram, juntas, nos anos de estudo, mais de $83 \%$ do total de ORSBCD, a saber, mais de nove mil mortes por ano. A diarreia pode ser causada pela falta de abastecimento de água, incluindo a falta de tratamento e o armazenamento inadequado da água no ambiente doméstico, além da ausência de esgotamento sanitário (UNICEF/WHO, 2009) e a doença de Chagas tem sua transmissão associada ao contato do homem suscetível com as

Tabela 1 - Óbitos totais, óbitos por causas definidas, óbitos por doenças infecciosas e parasitárias e óbitos por causas definidas relacionados ao saneamento básico inadequado. Brasil, 2001 a 2009.

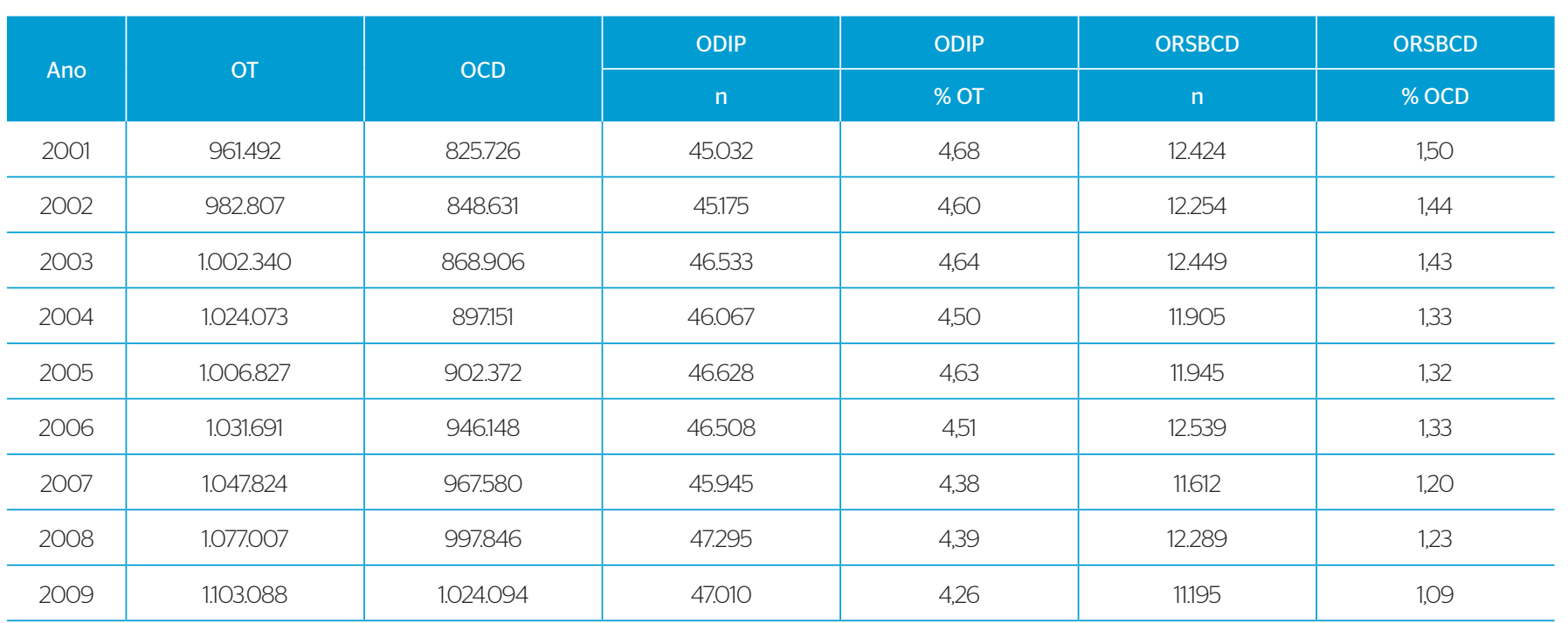

OT: óbitos totais; OCD: óbitos por causas definidas; ODIP: óbitos por doenças infecciosas e parasitárias; ORSBCD: óbitos por causas definidas relacionados ao saneamento básico inadequado.

Fonte: Sistema de Informações sobre Mortalidade (SIM/SUS), DATASUS (2011) 
Tabela 2 - Óbitos por causas não definidas, óbitos por causas não definidas relacionados ao saneamento básico inadequado, óbitos por causas definidas relacionadas ao saneamento básico inadequado, total de óbitos por doenças relacionadas ao saneamento básico inadequado. Brasil, 2001 a 2009.

\begin{tabular}{|c|c|c|c|c|c|}
\hline Ano & OCND & ORSBND & ORSBCD & ORSB & ORSB \\
\hline 2002 & 134.176 & 1.932 & 12.254 & 14.186 & 1,44 \\
\hline 2004 & 126.922 & 1.688 & 11.905 & 13.593 & 1,33 \\
\hline 2005 & 104.455 & 1.379 & 11.945 & 13.324 & 1,32 \\
\hline 2006 & 85.543 & 1.138 & 12.539 & 13.677 & 1,33 \\
\hline 2009 & 78.994 & 861 & 11.195 & 12.056 & 1,09 \\
\hline
\end{tabular}

OCND: óbitos por causas não definidas; ORSBND: óbitos por causas não definidas relacionados ao saneamento básico inadequado; ORSBCD: óbitos por causas definidas relacionadas ao saneamento básico inadequado; ORSB: total de óbitos por doenças relacionadas ao saneamento básico inadequado; OT: óbitos totais.

Fonte: Sistema de Informações sobre Mortalidade (SIM/SUS), DATASUS (2011).

Tabela 3 - Óbitos por causas definidas relacionados ao saneamento básico inadequado. Brasil, 2001 a 2009 - anos ímpares.

\begin{tabular}{|c|c|c|c|c|c|c|c|c|c|c|}
\hline Doenças & $\begin{array}{c}2001 \\
\text { óbitos }\end{array}$ & $\begin{array}{c}2001 \\
\%\end{array}$ & $\begin{array}{c}2003 \\
\text { óbitos }\end{array}$ & $\begin{array}{c}2003 \\
\%\end{array}$ & $\begin{array}{c}2005 \\
\text { óbitos }\end{array}$ & $\begin{array}{c}2005 \\
\%\end{array}$ & $\begin{array}{l}2007 \\
\text { óbitos }\end{array}$ & $\begin{array}{c}2007 \\
\%\end{array}$ & $\begin{array}{l}2009 \\
\text { óbitos }\end{array}$ & $\begin{array}{c}2009 \\
\%\end{array}$ \\
\hline Dengue & 42 & 0,34 & 69 & 0,55 & 48 & 0,40 & 332 & 2,86 & 375 & 3,35 \\
\hline Diarreias & 5.863 & 47,19 & 5.927 & 47,60 & 5.482 & 45,89 & 4.989 & 42,96 & 4.584 & 40,95 \\
\hline Doença de Chagas & 4.889 & 39,35 & 5.016 & 40,29 & 4.916 & 41,16 & 4.725 & 40,70 & 4.741 & 42,35 \\
\hline Esquistossomose & 583 & 4,69 & 464 & 3,73 & 514 & 4,30 & 534 & 4,60 & 498 & 4,45 \\
\hline Febre amarela & 7 & 0,06 & 7 & 0,06 & 1 & 0,01 & 5 & 0,04 & 13 & 0,12 \\
\hline Febres entéricas & 9 & 0,07 & 11 & 0,09 & 4 & 0,03 & 8 & 0,07 & 8 & 0,07 \\
\hline Hepatite A & 72 & 0,58 & 51 & 0,41 & 59 & 0,49 & 47 & 0,40 & 45 & 0,40 \\
\hline Leishmanioses & 220 & 1,17 & 247 & 1,98 & 261 & 2,19 & 305 & 2,63 & 360 & 3,21 \\
\hline Leptospirose & 388 & 3,12 & 341 & 2,74 & 337 & 2,82 & 391 & 3,37 & 329 & 2,94 \\
\hline Malária & 142 & 1,14 & 103 & 0,83 & 122 & 1,02 & 93 & 0,80 & 85 & 0,76 \\
\hline Teníases & 104 & 0,84 & 118 & 0,95 & 99 & 0,83 & 105 & 0,90 & 83 & 0,74 \\
\hline Tracoma & O & O & O & O & 1 & 0,01 & O & 0 & 0 & 0 \\
\hline
\end{tabular}

Fonte: Sistema de Informações sobre Mortalidade (SIM/SUS), DATASUS (2011). 
excretas de insetos conhecidos no Brasil como barbeiros, contaminadas pelo protozoário Trypanosoma cruzi (SILVEIRA, 2000). Entretanto,

a transmissão parece estar associada à densidade vetorial e à resistência do hospedeiro, o que poderia explicar o fato de $30 \%$ de indivíduos residentes em áreas de alta infestação permanecerem soronegativos (GONTIJO \& SANTOS, 2011).

Como terceira causa de ORSBCD aparece a esquistossomose, que tem sua transmissão associada à água. Porém, uma percentagem muito pequena da população brasileira atribuiu aos banhos de rio e às lavagens de roupas nestes o papel da transmissão da esquistossomose (RIBEIRO, 2004).

A doença de Chagas, que foi responsável por aproximadamente $40 \%$ das mortes por óbitos por causas definidas relacionadas ao saneamento básico inadequado, teve $68 \%$ dos óbitos como vítimas indivíduos com mais de 60 anos, confirmando que a proporção de óbitos é, em geral, crescente conforme a idade (Guariento et al., 2011).

\section{Morbidade ambulatorial Sistema Único de Saúde por doenças de notificação compulsória relacionadas ao saneamento básico inadequado}

Entre as doenças relacionadas ao saneamento básico inadequado, atendidas no SUS, foram selecionadas aquelas que são de notificação compulsória no País segundo a Portaria GM/MS nº 104, de 25 de janeiro de 2011 (BRASIL, 2011), a saber: cólera, dengue, doença de Chagas aguda, esquistossomose, febre amarela, febre tifoide, hepatite, leishmaniose visceral, leptospirose e malária. A Tabela 4 apresenta o número de casos notificados no SUS destas doenças (MADRSB) e a taxa anual de incidência (TI) para os anos ímpares do período de 2001 a 2009.

Entre as dez doenças de MADRSB atendidas no SUS, a média anual foi de 466.351 casos. As quatro doenças com maior número de casos foram, pela ordem, a dengue, a hepatite, a esquistossomose e a leptospirose.

No período entre 2001 e 2009, a taxa de incidência média de dengue foi de 186,58 casos da doença por 100 mil habitantes. As regiões com as maiores taxas de incidência da doença no período de estudo foram as regiões Centro-Oeste (312,55 casos por 100 mil habitantes) e Nordeste (257,56 casos por 100 mil habitantes). Porém, a região com maior número de casos foi a região Sudeste com mais de 1,5 milhões de casos entre 2001 e 2009.

A hepatite apresentou taxa média de incidência correspondente a 27,25 casos por 100 mil habitantes, com maior proporção de casos na região Sudeste. A esquistossomose apresentou taxa de incidência média de 22,63 casos por 100 mil habitantes, principalmente com infestação endêmica em partes das regiões Nordeste, Centro-Oeste e norte do Estado de Minas Gerais. Já a leptospirose, com taxa média de incidência de 1,92 casos, tem o maior número de notificações na região Sudeste.

Em relação ao total de casos de doenças de notificação compulsória relacionadas ao saneamento básico inadequado, as regiões com maiores taxas de incidência foram a Região Centro-Oeste (796,96 casos por 100 mil habitantes) e Norte (383,82 casos por 100 mil habitantes). Por outro lado, a região com menor incidência foi a região Sul (46,73 casos por 100 mil habitantes).

Tabela 4 - Casos de doenças de notificação compulsória relacionados ao saneamento básico inadequado e taxa de incidência anual. Brasil, 2001 a 2009 - anos ímpares.

\begin{tabular}{|c|c|c|c|c|c|c|c|c|c|c|}
\hline Doenças & $\begin{array}{c}2001 \\
\text { óbitos }\end{array}$ & $\begin{array}{c}2001 \\
\mathrm{TI}\end{array}$ & $\begin{array}{c}2003 \\
\text { óbitos }\end{array}$ & $\begin{array}{c}2003 \\
\text { TI }\end{array}$ & $\begin{array}{l}2005 \\
\text { óbitos }\end{array}$ & $\begin{array}{c}2005 \\
\text { TI }\end{array}$ & $\begin{array}{l}2007 \\
\text { óbitos }\end{array}$ & $\begin{array}{c}2007 \\
\text { TI }\end{array}$ & $\begin{array}{l}2009 \\
\text { óbitos }\end{array}$ & $\begin{array}{c}2009 \\
\text { TI }\end{array}$ \\
\hline Cólera & 7 & 0,00 & $\mathrm{O}$ & 0,00 & 5 & 0,00 & 0 & 0,00 & $\mathrm{O}$ & 0,00 \\
\hline Dengue & 386.860 & 224,42 & 279.139 & 157,82 & 151.047 & 83,29 & 500.339 & 269,38 & 386.133 & 203,24 \\
\hline $\begin{array}{l}\text { Doença de } \\
\text { Chagas aguda }\end{array}$ & 59 & 0,03 & 579 & 0,33 & 629 & 0,35 & 155 & 0,08 & 254 & 0,13 \\
\hline Esquistossomose & 45.998 & 26,68 & 53.047 & 29,99 & 47.300 & 26,08 & 33.203 & 17,88 & 18.097 & 9,53 \\
\hline Febre amarela & 35 & 0,02 & 55 & 0,03 & 5 & 0,00 & 5 & 0,00 & 50 & 0,03 \\
\hline Febre entéricas & 380 & 0,22 & 869 & 0,49 & 494 & 0,27 & 404 & 0,22 & 305 & 0,16 \\
\hline Hepatite & 43.536 & 25,25 & 41.442 & 23,43 & 64.324 & 35,47 & 49.368 & 26,58 & 47.143 & 24,81 \\
\hline Leishmaniose & 2.860 & 1,66 & 3.344 & 1,89 & 3.850 & 2,12 & 3.562 & 1,92 & 3.892 & 2,05 \\
\hline Leptospirose & 3.679 & 2,13 & 3.050 & 1,72 & 3.464 & 1,91 & 3.292 & 1,77 & 3.941 & 2,07 \\
\hline Malária & 262 & 0,15 & 1.076 & 0,61 & 1.686 & 0,93 & 1.173 & 0,63 & 855 & 0,45 \\
\hline Total & 483.676 & 280,58 & 382.601 & 216,31 & 272.804 & 150,44 & 591.501 & 318,46 & 460.670 & 242,47 \\
\hline
\end{tabular}

TI: taxa de incidência - casos por 100 mil habitantes.

Fonte: Sistema de Informação de Agravos de Notificação (SinanWeb/SUS), DATASUS (2011) 


\section{Morbidade hospitalar Sistema Único} de Saúde por doenças relacionadas ao saneamento básico inadequado

$\mathrm{Na}$ Tabela 5, é apresentada a morbidade hospitalar no SUS por doenças relacionadas ao saneamento básico inadequado (MHDRSB) no período de 2001 a 2009, a saber, anos ímpares do período de estudo.

A média de internações hospitalares no SUS por MHDRSB no período de 2001 a 2009 foi de 758.750 internações por ano. Segundo a Tabela 5, o número de internações hospitalares no SUS por estas doenças reduziu 5,72\% nos nove anos do estudo, sendo que a diarreia e a dengue foram responsáveis por mais de $93 \%$ das internações hospitalares por MHDRSB no SUS entre os anos de 2003 e 2009.

A morbidade hospitalar por doenças relacionadas ao saneamento básico inadequado tem maiores proporções de internações anuais nas regiões Norte (7,50 internações por 1.000 habitantes) e Nordeste (6,20 internações por 1.000 habitantes) e com menor proporção de internações por ano na região Sudeste (1,71 internações por 1.000 habitantes).

\section{Gastos do Sistema Único de Saúde com atendimento ambulatorial devido a doenças de notificação compulsória relacionadas ao saneamento básico inadequado}

No período entre 2001 e 2009, verificou-se a realização de 4.197.160 atendimentos ambulatoriais devido a doenças de notificação compulsória relacionadas ao saneamento básico inadequado (Consultas DRSB). Neste período, o valor pago pelo SUS por consulta médica variou de um valor mínimo de $\mathrm{R} \$ 2,55$, em 2001, a um valor máximo de $\mathrm{R} \$ 10,00$, nos anos 2008 e 2009. Para calcular o gasto do SUS com atendimento ambulatorial devido a doenças de notificação compulsória relacionadas ao saneamento básico inadequado (GADRSB), multiplicou-se o número de consultas anual pelo valor unitário do procedimento ambulatorial pago pelo SUS.

A seguir, buscou-se junto ao SIA/SUS o valor total gasto pelo SUS com consultas médicas para cada ano do estudo (GATSUS). Assim, pôde-se observar na Tabela 6 que o gasto do SUS com atendimento ambulatorial devido a doenças de notificação compulsória relacionadas ao saneamento básico oscilou entre $0,11 \%$ e $0,59 \%$ do valor total pago com consultas médicas no SUS. Ainda, no período em estudo, o gasto anual do SUS com consultas médicas devido a doenças de notificação compulsória relacionadas ao saneamento básico inadequado foi, na média, de $0,26 \%$ do gasto total com consultas médicas.

\section{Gastos do Sistema Único de Saúde com morbidade hospitalar por doenças relacionadas ao saneamento básico inadequado}

Para as doenças relacionadas com deficiências do saneamento básico (DRSB), os valores absolutos pagos pelas internações (GIDRSB) cresceram 141,4\% no período de estudo, passando de R $148.729 .323,95$

Tabela 5 - Morbidade hospitalar Sistema Único de Saúde por doenças relacionadas ao saneamento básico inadequado. Brasil, 2001 a 2009 - anos ímpares.

\begin{tabular}{|c|c|c|c|c|c|c|c|c|c|c|}
\hline Doenças & $\begin{array}{c}2001 \\
\text { Óbitos }\end{array}$ & $\begin{array}{c}2001 \\
\%\end{array}$ & $\begin{array}{l}2003 \\
\text { óbitos }\end{array}$ & $\begin{array}{c}2003 \\
\%\end{array}$ & $\begin{array}{l}2005 \\
\text { óbitos }\end{array}$ & $\begin{array}{c}2005 \\
\%\end{array}$ & $\begin{array}{l}2007 \\
\text { óbitos }\end{array}$ & $\begin{array}{c}2007 \\
\%\end{array}$ & $\begin{array}{l}2009 \\
\text { óbitos }\end{array}$ & $\begin{array}{c}2009 \\
\%\end{array}$ \\
\hline Dengue & 24.226 & 0,19 & 54.396 & 6,65 & 32.432 & 4,25 & 53.461 & 7,65 & 54.482 & 7,63 \\
\hline Doença de Chagas & 1.301 & 0,17 & 3.696 & 0,45 & 2.392 & 0,31 & 1.467 & 0,21 & 754 & 0,11 \\
\hline Doenças de pele & 5.126 & 0,68 & 5.820 & 0,71 & 5.339 & 0,68 & 5.677 & 0,81 & 10.872 & 1,52 \\
\hline Esquistossomose & 1.267 & 0,17 & 1.017 & 0,12 & 890 & 0,12 & 722 & 0,10 & 366 & 0,05 \\
\hline Febre amarela & 48 & 0,01 & 79 & 0,01 & 21 & 0,01 & 16 & 0,01 & 62 & 0,01 \\
\hline Febres entéricas & 2.775 & 0,37 & 1.185 & 0,15 & 886 & 0,12 & 627 & 0,09 & 1.145 & 0,16 \\
\hline Filariose & 98 & 0,01 & 200 & 0,01 & 82 & 0,01 & 89 & 0,01 & 302 & 0,01 \\
\hline Helmintíases & 1.175 & 0,15 & 1.443 & 0,18 & 1.207 & 0,16 & 1.209 & 0,17 & 906 & 0,13 \\
\hline Leptospirose & 3.689 & 0,49 & 3.016 & 0,37 & 3.099 & 0,41 & 2.975 & 0,43 & 2.591 & 0,36 \\
\hline Malária & 14.751 & 1,94 & 10.690 & 1,31 & 12.542 & 1,64 & 6.772 & 0,96 & 4.705 & 0,66 \\
\hline Teníases & 566 & 0,07 & 572 & 0,07 & 600 & 0,07 & 575 & 0,08 & 692 & 0,09 \\
\hline Tracoma & 8 & 0,01 & 3 & 0,01 & 3 & 0,01 & 5 & 0,01 & 3 & 0,01 \\
\hline Total & 758.776 & 100,00 & 818.348 & 100,00 & 763.275 & 100,00 & 698.836 & 100,00 & 715.384 & 100,00 \\
\hline
\end{tabular}


em 2001 para R\$ 359.040.900,09 em 2009; valores que correspondem, respectivamente, a 2,92 e 3,55\% do gasto total do SUS com internações hospitalares no país (Tabela 7). Em valores relativos, o gasto com internações hospitalares devido a doenças relacionadas ao saneamento básico inadequado (GIDRSB) em relação ao gasto total do SUS com internações hospitalares no país (GITSUS) aumentou 21,58\% durante os anos da pesquisa, sendo o gasto médio anual com internações hospitalares devido a doenças relacionadas ao saneamento básico inadequado foi de 3,33\% do gasto total do SUS com internações hospitalares no período de estudo.

$\mathrm{O}$ ano com maior número de internações hospitalares devido a doenças relacionadas ao saneamento básico inadequado foi 2003, com 818.759 internações e o ano com o menor número de internações devido a tais doenças foi o ano de 2007, com 699.136 internações conforme pode ser observado na Tabela 7 .

Tabela 6 - Gastos do Sistema Único de Saúde com atendimento ambulatorial devido a doenças de notificação compulsória relacionadas ao saneamento básico inadequado e com todas as doenças. Brasil, 2001 a 2009.

\begin{tabular}{c|c|c|c|c|c} 
Ano de estudo & Consultas DRSB (número) & Valor unitário (R\$) & Valor GADRSB (R\$) & Valor total GATSUS (R\$) & GADRSB/GATSUS (\%) \\
\hline 2001 & 483.676 & 2,55 & $1.233 .373,80$ & $530.727 .328,87$ & 0,23 \\
\hline 2002 & 802.852 & 5,05 & $4.054 .402,60$ & $681.769 .806,25$ & 0,59 \\
\hline 2003 & 382.601 & 7,55 & $2.888 .637,55$ & $1.049 .514 .537,42$ & 0,28 \\
\hline 2004 & 185.244 & 7,55 & $1.398 .592,20$ & $1.315 .593 .578,47$ & 0,11 \\
\hline 2005 & 272.804 & 7,55 & $2.059 .670,20$ & $1.420 .814 .413,01$ & 0,14 \\
\hline 2006 & 384.504 & 7,55 & $2.903 .005,20$ & $1.533 .858 .766,90$ & 0,19 \\
\hline 2007 & 591.501 & 8,37 & $4.950 .863,37$ & $1.728 .769 .217,84$ & 0,29 \\
\hline 2008 & 633.308 & 10 & $6.333 .080,00$ & $1.948 .447 .323,22$ & 0,33 \\
\hline 2009 & 460.670 & 10 & $4.606 .700,00$ & $2.196 .040 .357,61$ & 0.21 \\
\hline Total & 4.197 .160 & - & $30.428 .324,92$ & $12.405 .535 .329,59$ & - \\
\hline
\end{tabular}

DRSB: doenças relacionadas com deficiências do saneamento básico; GADRSB: Gastos do Sistema Único de Saúde com atendimento ambulatorial devido a doenças de notificação compulsória relacionadas ao saneamento básico inadequado; GATSUS: gastos com todas as doenças.

Fonte: Sistema de Informações Ambulatoriais do SUS (SIA/SUS), DATASUS (2011).

Tabela 7 - Gastos com internações hospitalares devido a doenças relacionadas ao saneamento básico inadequado e com todas as doenças. Brasil, 2001 a 2009.

\begin{tabular}{c|c|c|c|c|c} 
Ano de estudo & Internações DRSB (número) & Valor médio (R\$) & Valor GIDRSB (R\$) & Valor total GITSUS (R\$) & GIDRSB/GITSUS (\%) \\
\hline 2001 & 759.077 & 195,93 & $148.729 .323,95$ & $5.096 .155 .505,04$ & 2,92 \\
\hline 2002 & 799.985 & 219,47 & $175.570 .508,53$ & $5.406 .391 .841,17$ & 3,25 \\
\hline 2003 & 818.759 & 251,92 & $206.262 .047,61$ & $5.861 .712 .487,36$ & 3,52 \\
\hline 2004 & 729.010 & 299,68 & $218.467 .946,59$ & $6.581 .123 .333,54$ & 3,32 \\
\hline 2005 & 763.500 & 306,57 & $234.065 .046,29$ & $6.956 .764 .584,29$ & 3,36 \\
\hline 2006 & 775.018 & 295,49 & $229.288 .002,94$ & $6.998 .043 .548,10$ & 3,28 \\
\hline 2007 & 699.136 & 312,34 & $218.369 .438,61$ & $7.617 .769 .441,99$ & 2,89 \\
\hline 2008 & 771.083 & 417,30 & $321.774 .420,00$ & $8.286 .055 .941,30$ & 3,80 \\
\hline 2009 & 715.384 & 501,89 & $359.040 .900,09$ & $10.124 .918 .629,27$ & 6 \\
\hline Total & 6.830 .952 & - & $2.111 .567 .634,61$ & $62.928 .935 .312,06$ & 3,55 \\
\hline
\end{tabular}

DRSB: doenças relacionadas com deficiências do saneamento básico; GIDRSB: gastos com internações hospitalares devido a doenças relacionadas ao saneamento básico inadequado: GITSUS: gastos com todas as doenças.

Fonte: Sistema de Informações Hospitalares do SUS (SIH/SUS), DATASUS (2011) 


\section{CONCLUSÃO}

O presente artigo mostrou que as doenças relacionadas ao saneamento básico inadequado foram responsáveis, em média, por 13.449 óbitos por ano ao longo do período de 2001 a 2009, 1,31\% dos óbitos ocorridos no período. As diarreias e a doença de Chagas representaram mais de $83 \%$ do total de óbitos por causas definidas relacionadas ao saneamento básico inadequado, ou seja, mais de nove mil mortes por ano.

As quatro doenças de notificação compulsória associadas a deficiências ou inexistência de saneamento básico com maior número de casos notificados no período foram, pela ordem, a dengue, a hepatite, a esquistossomose e a leptospirose. No período de estudo, ocorreram, em média, 466.351 consultas de doenças de notificação compulsória e 758.750 internações hospitalares por ano.

Todos esses agravados levaram a uma despesa total de 2,141 bilhões de reais no período 2001 a 2009, ou seja, 2,84\% do gasto total do SUS com consultas médicas e internações hospitalares.

Este quadro de saúde pública revela uma herança de sucessivas políticas de saneamento básico, em que os municípios viram-se à margem do acesso a recursos para investimentos e de oportunidades para constituição de sua capacidade técnica e gerencial. Viu-se também comprometida a prestação de serviços públicos de saneamento básico sob a ótica da universalidade e integralidade, tendo como resultado um quadro de doenças associadas ao saneamento básico incompatível com o grau de desenvolvimento atingido pelo país.

\section{REFERÊNCIAS}

BRASIL. Presidência da República. Casa Civil (2007). Lei n 11.445, de 5 de janeiro de 2007. Estabelece diretrizes nacionais para 0 saneamento básico; altera as Leis $n^{\circ} 6.766$, de 19 de dezembro de $1979,8.036$, de 11 de maio de 1990, 8.666, de 21 de junho de 1993, 8.987, de 13 de fevereiro de 1995; revoga a Lei n 6.528, de 11 de maio de 1978 e dá outras providências. Diário Oficial da União, 8 jan. 2007 [retificado no DOU de 11 jan. 2007].

BRASIL. Ministério das Cidades; Berenice de Souza Cordeiro (Coord.) (2009) Secretaria Nacional de Saneamento Ambiental. Programa de Modernização do Setor Saneamento (PMSS). Instrumentos das políticas e da gestão dos serviços públicos de saneamento básico. Brasília: Ministério das Cidades.

BRASIL. Ministério da Saúde (2011). Portaria GM/MS No 104, de 25 de janeiro de 2011. Define as terminologias adotadas em legislação nacional, conforme o disposto no Regulamento Sanitário Internacional 2005 (RSI 2005), a relação de doenças, agravos e eventos em saúde pública de notificação compulsória em todo o território nacional e estabelece fluxo, critérios, responsabilidades e atribuições aos profissionais e serviços de saúde. Diário Oficial da União, 26 jan. 2011.

CARVALHO, D.M. (1997) Grandes sistemas nacionais de informações de saúde: revisão e discussão da situação atual. Informe Epidemiológico do SUS, v. 4. Brasília: Ministério da Saúde.

COSTA, A.M.; PONTES, C.A.A.; GONÇALVES, F.R.; LUCENA, R.C.B.; CASTRO, C.C.L.; GALINDO, E.F.; MANSUR, M.C. (2010) Impactos na saúde e no Sistema Único de Saúde decorrentes de agravos relacionados a um saneamento ambiental inadequado. In: Fundação Nacional de Saúde. Primeiro caderno de pesquisa em engenharia de saúde pública. Brasília: Fundação Nacional de Saúde, p. 7-27.
DANIEL, L.A.; BRANDÃO, C.S.S.; GUIMARÃES, J.R.; LIBÂNIO, M.; DE LUCA, S. (2001) Processos de desinfecção e desinfetantes alternativos na produção de água potável. Rio de Janeiro: RiMa, ABES.

DATASUS - Departamento de Informática do Sistema Único de Saúde. (2011) Indicadores e Dados Básicos de Saúde - Brasil 2011. Disponível em: <http://www2.datasus.gov.br/DATASUS/ index.php?area=02>. Acesso em: 01 jul. 2011.

GONTIJO, E.D.; SANTOS, S.E. (2011) Mecanismos principais e atípicos de transmissão da doença de Chagas. Disponível em: $\quad<$ http://www.fiocruz.br/chagas/cgi/cgilua.exe/sys/start. htm?sid=25>. Acesso em: 31 out. 2011.

GUARIENTO, M.E.; CARRIJO, C.M.; ALMEIDA, E.A.; MAGNA, L.A. (2011) Perfil clínico de idosos portadores de doença de Chagas atendidos em serviço de referência. Revista da Sociedade Brasileira de Clínica Médica, v. 9, n. 1, p. 20-24.

IBGE - Instituto Brasileiro de Geografia e Estatística. (2010) Pesquisa Nacional de Saneamento Básico - 2008. Disponível em: <http://www.ibge.gov.br/home/presidencia/noticias/noticia visualiza.php?id_noticia $=1691 \& i d \_p a g i n a=1>$. Acesso em: $2 \overline{0}$ ago. 2010.

IBGE - Instituto Brasileiro de Geografia e Estatística. (2011) Primeiros resultados definitivos do Censo 2010. Disponível em: <http://www. ibge.gov.br/home/presidencia/noticias/noticia_visualiza.php?id_ noticia=1866\&id_pagina=1 > . Acesso em: 29 abr. 2011.

OMS - Organização Mundial de Saúde. (1997) Classificação estatística internacional de doenças e problemas relacionados à saúde. 10. rev. São Paulo: USP/OPAS. 
OMS - Organização Mundial dA Saúde. (2007) Doenças ambientais matam 233 mil por ano no Brasil. Disponível em: <http://www. bbc.co.uk/portuguese/reporterbbc/story/2007/06/070613_oms_ doencas_pu.shtml>. Acesso em: 13 jun. 2007.

OMS - Organização Mundial dA Saúde. (2010) Relatório Mundial da Saúde. Financiamento dos Sistemas de Saúde: O caminho para cobertura universal. Genebra: OMS.

OPAS - Organização Pan-Americana de Saúde. (2007) Saúde nas Américas: 2007 - v1 Regional. Washington: OPAS.
RIBEIRO, P.J. (2004) Programa educativo em esquistossomose: modelo de abordagem metodológica. Revista de Saúde Pública, v. 38, n. 3, p. 415-421.

SILVEIRA, A.C. (2000) Situação do controle da transmissão vetorial da doença de Chagas nas Américas. Cadernos de Saúde Pública, v. 16 , sup. 2 , p. $35-42$.

UNICEF/WHO - UNITED NATIONS CHILDREN'S FUND/WORLD HEALTH ORGANIZATION. (2009) Diarrhoea: why children are still dying and what can be done. Geneva: UNICEF/WHO. 